\title{
Underlying heart diseases and acute COVID-19 outcomes
}

Iván J. Núñez-Gil ${ }^{1,2}$, Antonio Fernández-Ortiz, ${ }^{1,2}$, Charbel Maroun Eid ${ }^{3}$, Jia Huang ${ }^{4}$, Rodolfo Romero ${ }^{5}$, Víctor Manuel Becerra-Muñoz ${ }^{6}$, Aitor Uribarri ${ }^{7}$, Gisela Feltes ${ }^{8}$,

Daniela Trabattoni ${ }^{9}$, Inmaculada Fernández-Rozas ${ }^{10}$, María C. Viana-Llamas ${ }^{11}$, Martino Pepe ${ }^{12}$, Enrico Cerrato ${ }^{13}$, Maurizio Bertaina ${ }^{14}$, Thamar Capel Astrua ${ }^{15}$, Emilio Alfonso $^{16}$, Alex F. Castro-Mejía ${ }^{17}$, Sergio Raposeiras-Roubin ${ }^{18}$, Fabrizio D’Ascenzo $^{19}$, Carolina Espejo Paeres ${ }^{20}$, Jaime Signes-Costa ${ }^{21}$, Alfredo Bardají ${ }^{22}$, Cristina Fernandez-Pére $z^{1,2,23}$, Francisco Marín ${ }^{24}$, Oscar Fabregat-Andres ${ }^{25}$, Ibrahim Akin ${ }^{26}$, Vicente Estrada ${ }^{1,2}$, Carlos Macaya ${ }^{1,2}$; on behalf of HOPE COVID-19 Investigators

${ }^{1}$ Hospital Clínico San Carlos, Universidad Complutense de Madrid, Instituto de Investigación Sanitaria del Hospital Clínico San Carlos (IdISSC), Madrid, Spain; ${ }^{2}$ Universidad Complutense de Madrid, Spain; ${ }^{3}$ Hospital Universitario La Paz, Instituto de Investigación Hospital Universitario La Paz (IdiPAZ), Madrid, Spain; ${ }^{4}$ The Second People's Hospital of Shenzhen, China; ${ }^{5}$ Hospital Universitario Getafe, Madrid, Spain and Universidad Europea de Madrid, Spain; ${ }^{6}$ Unidad de Gestión Clínica Área del Corazón, Instituto de Investigación Biomédica de Málaga (IBIMA), Hospital Universitario Virgen de la Victoria, Universidad de Málaga (UMA), Centro de Investigación Biomédica en Red de Enfermedades Cardiovasculares (CIBERCV), Málaga, Spain; ${ }^{7}$ CIBER-CV, Hospital Clínico Universitario de Valladolid, Valladolid, Spain; ${ }^{8}$ Hospital Nuestra Señora de América, Madrid, Spain; ${ }^{9}$ Centro Cardiologico Monzino, IRCCS, Milano, Italy;

${ }^{10}$ Hospital Severo Ochoa, Leganés, Spain; ${ }^{11}$ Hospital Universitario Guadalajara, Spain; ${ }^{12}$ Azienda Ospedaliero-Universitaria Consorziale Policlinico di Bari, Italy; ${ }^{13}$ San Luigi Gonzaga University Hospital, Rivoli, Turin, Italy; ${ }^{14}$ Martini Hospital, Emergency Medicine Department Turin, Italy; ${ }^{15}$ Hospital Virgen del Mar, Madrid, Spain; ${ }^{16}$ Instituto de Cardiología y Cirugía Cardiovascular, Havana, Cuba; ${ }^{17}$ Hospital General del Norte de Guayaquil IESS Los Ceibos, Guayaquil, Ecuador; ${ }^{18}$ Hospital Universitario Álvaro Cunqueiro, Instituto de Investigación Sanitaria Galicia Sur, Vigo, Spain; ${ }^{19}$ San Giovanni Battista Hospital, Turin, Italy; ${ }^{20}$ Hospital Universitario Príncipe de Asturias, Alcalá de Henares, Spain; ${ }^{21}$ Hospital Clínico Universitario, Incliva, Universidad de Valencia, Spain; ${ }^{22}$ University Hospital Joan XXIII, University Rovira

Virgili, IISPV, Tarragona, Spain; ${ }^{23}$ Fundación Instituto para la Mejora de la Asistencia Sanitaria (IMAS),

Madrid, Spain; ${ }^{24}$ Hospital Clínico Universitario Virgen de la Arrixaca, IMIB-Arrixaca, Universidad de Murcia,

CIBERCV, Murcia, Spain; ${ }^{25}$ Hospital IMED, Valencia, Spain; ${ }^{26}$ First Department of Medicine, Medical

Faculty Mannheim, University Heidelberg, Mannheim, Germany and DZHK (German Center for

Cardiovascular Research), Partner Site, Heidelberg-Mannheim, Mannheim, Germany

\section{Abstract}

Background: The presence of any underlying heart condition could influence outcomes during the coronavirus disease 2019 (COVID-19).

Methods: The registry HOPE-COVID-19 (Health Outcome Predictive Evaluation for COVID-19, NCT04334291) is an international ambispective study, enrolling COVID-19 patients discharged from hospital, dead or alive.

Address for correspondence: Iván Javier Núñez-Gil, MD, PhD, MSc, Hospital Clínico San Carlos, Prof. Martin Lagos St. 28040, Madrid, Spain, tel: 0034 913303283, fax: 0034 913303730, e-mail: ibnsky@yahoo.es 
Results: HOPE enrolled 2798 patients from 35 centers in 7 countries. Median age was 67 years (IQR: 53.0-78.0), and most were male (59.5\%). A relevant heart disease was present in 682 (24\%) cases. These were older, more frequently male, with higher overall burden of cardiovascular risk factors (hypertension, dyslipidemia, diabetes mellitus, smoking habit, obesity) and other comorbidities such renal failure, lung, cerebrovascular disease and oncologic antecedents ( $p<0.01$, for all). The heart cohort received more corticoids (28.9\% vs. 20.4\%, $p<0.001$ ), antibiotics, but less hydroxychloroquine, antivirals or tocilizumab. Considering the epidemiologic profile, a previous heart condition was independently related with shortterm mortality in the Cox multivariate analysis (1.62; 95\% CI 1.29-2.03; $p<0.001$ ). Moreover, heart patients needed more respiratory, circulatory support, and presented more in-hospital events, such heart failure, renal failure, respiratory insufficiency, sepsis, systemic infammatory response syndrome and clinically relevant bleedings (all, $p<0.001$ ), and mortality (39.7\% vs. 15.5\%; $p<0.001$ ).

Conclusions: An underlying heart disease is an adverse prognostic factor for patients suffering COVID-19. Its presence could be related with different clinical drug management and would benefit from maintaining treatment with angiotensin converting enzyme inhibitors or angiotensin receptor blockers during in-hospital stay.

Trial Numbers: NCT04334291/EUPAS34399. (Cardiol J 2021; 28, 2: 202-214)

Key words: COVID-19, mortality, cardiology, registry, prognosis, heart disease

\section{Introduction}

The recent outbreak of a zoonotic viral disease named coronavirus disease 2019 (COVID-19) [1] has been declared a pandemic by World Health Organization (WHO) [2]. With important morbimortality [3], some early-published data have already pointed-out previous or underlying heart conditions to be at higher risk for worse outcomes $[4,5]$. Moreover, according to the American Centers for Disease Control and Prevention, elderly patients with comorbidities are at higher risk of becoming infected with COVID-19, especially those with coronary heart disease, hypertension, or diabetes [6]. In fact, some authors have suggested that the mortality rate of this respiratory-borne coronavirus or severe acute respiratory syndrome coronavirus 2 (SARS-CoV-2) could be even higher in those with previous cardiovascular diseases than in patients with previous chronic respiratory diseases [7]. Furthermore, this is relevant since an important percentage of patients admitted for COVID-19 will present with an underlying cardiac problem. In a recent Chinese series, 25\% had heart diseases, $44 \%$ had arrhythmias, and $58 \%$ had hypertension [8]. Additionally, there is preliminary evidence suggesting that the responsible virus affects primarily the cardiovascular system and the heart itself with direct myocardial injury among other deleterious mechanisms $[9,10]$.

Taken together, to sum up, there is growing evidence that underlying cardiovascular conditions lead to a higher likelihood of COVID-19 infection, more severe disease progression, and higher risk for mortality [11]. Moreover, the pandemic has posed a major impact in the treatment of regular heart diseases [12].

We analyze herein, the clinical profile, presentation and influence of previous treatments, primarily focusing on the mortality of patients with any underlying heart condition hospitalized because of COVID-19.

\section{Methods}

The present study was approved by the ethics committee of the promoting center, and was appraised and accepted by institutional board or local committees as well. Written informed consent was waived because of its anonymized observational design. All local principal researchers reviewed the draft and vouch for the accuracy and veracity of data. A complete list of hospitals, investigators, collaborators and definitions is available in the

\section{Supplementary Appendix.}

\section{Study design and participation criteria}

The registry HOPE-COVID-19 (Health Outcome Predictive Evaluation for COVID-19, NCT04334291) is an international investigatorinitiated study without conflicts of interest [13]. It was designed as an ambispective cohort, real life all-comers type, without any financial remuneration for researchers. Patients were eligible for enrollment when discharged after an in-hospital admission with a positive COVID-19 test or if their 
attending physicians considered them highly likely to have presented the infection. Confirmed cases were those with positive throat swab samples tested using real-time reverse transcriptase-polymerase chain reaction assays according to the WHO recommendations. All decisions and clinical procedures were performed by the attending physician team independently of this study following the local regular practice and protocols. The data was collected in electronic format in a secure online database (www. HopeProjectMD.com). The information presented here correspond to the HOPE COVID-19 Registry with a cutoff performed on April 18 $8^{\text {th }}, 2020$.

\section{Definitions, objectives and study outcomes}

A pragmatic definition of heart disease was adopted and divided into various groups according to the local research team, led by two experienced physicians. Any heart disease was considered when it was stated in the clinical history and/or the patient was receiving medication for that purpose. The following categories for the main heart problem of every patient were accepted: arrhythmias, coronary artery disease, heart failure or cardiomyopathy, heart valve disease, combined (when various of the former problems were present to a clinically relevant degree) and non-specified or other different from the mentioned groups (i.e. congenital heart disease). Study definitions are available in the appendix and online in the study webpage.

The objectives were:

- comparing the epidemiological and clinical profiles and management of COVID-19 patients with vs. without previous heart disease;

- determining the prognostic impact of an underlying heart disease on mortality;

- identifying independent predictors of mortality in the group with underlying heart disease.

The reference primary end-point was considered all-cause mortality. Other events were recorded as secondary end-points, such as invasive mechanical ventilation, non-invasive mechanical ventilation, prone, respiratory insufficiency, heart failure, renal failure, upper respiratory tract involvement, pneumonia, sepsis, systemic inflammatory response syndrome, clinically relevant bleeding, hemoptysis and embolic events. Events were allocated following local researcher criteria upon HOPE COVID-19 registry definitions.

\section{Statistical analysis}

Data are presented as mean \pm standard deviation for continuous variables with a normal distribution, median (interquartile range $[\mathrm{IQR}]$ ) for continuous

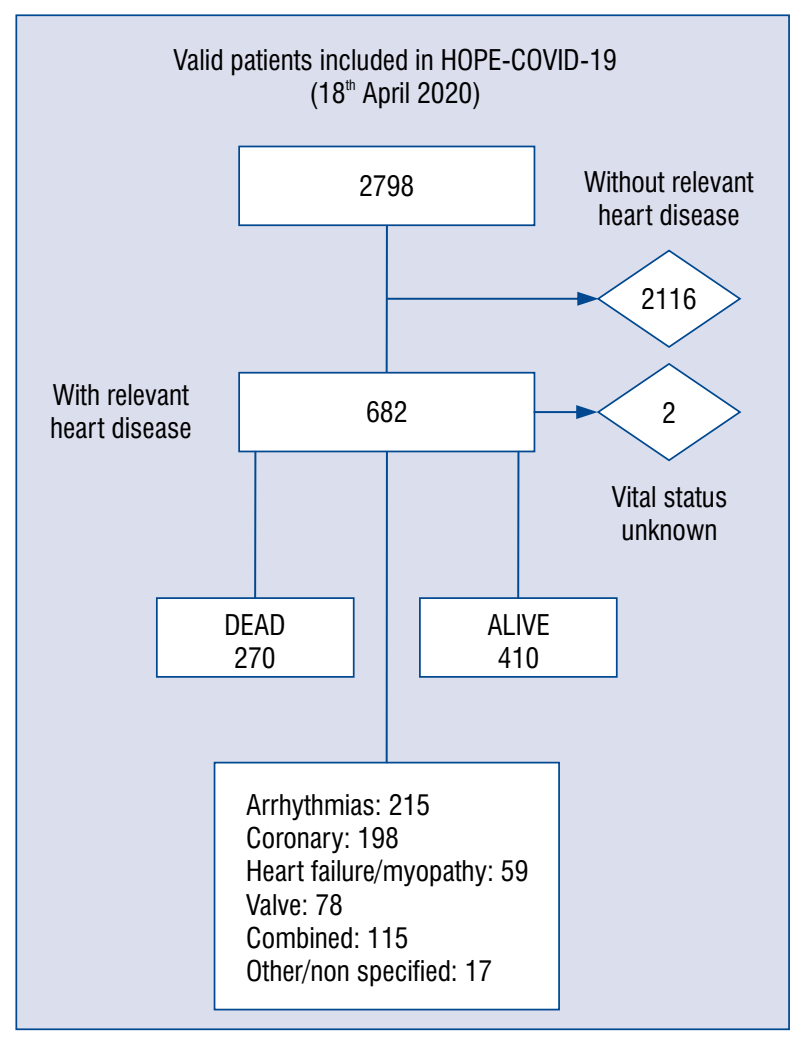

Figure 1. Study flow diagram.

variables with a non-normal distribution, and as frequency (\%) for categorical variables. The Student t-test and the Mann-Whitney U-test were used to compare continuous variables with normal and nonnormal distributions, when needed. The Chi-squaredtest was used to compare categorical variables. Given the multiplicity of variables, only factors with $\mathrm{p}<0.05$ on the mentioned univariate analysis were entered into the Cox multivariate analysis. Mortality analysis was performed using the Kaplan-Meier estimates and log-rank tests to compare factors. Statistical analysis was completed with SPSS statistics v24.0 (SPSS, Inc., Chicago, IL, USA) in all analyses. All tests were two-sided, and a p-value less than 0.05 was considered statistically significant.

\section{Results}

Finally, 2798 patients were enrolled in HOPE registry up to $18^{\text {th }}$ April, 2020, from 35 centers in 25 cities and 7 countries (Canada, China, Cuba, Ecuador, Germany, Italy, and Spain) (Fig. 1).

\section{Epidemiologic and clinical profiles}

The median age was 67 years (IQR 53.0-78.0), and most were male (60\%). The most frequent co- 
morbidities were hypertension (49\%) and dyslipidemia (35.1\%). A relevant heart disease [13] was recorded in 682 (24\%) cases. Further details are displayed, stratified by the presence of any heart disease or not, in Table 1 . In the heart-disease cohort, deceased heart patients were older $(\mathrm{p}<$ $<0.001)$ and more frequently male $(58.5 \%$ vs. $65.8 \%, \mathrm{p}=0.001$ ), with a higher overall burden of cardiovascular risk factors (hypertension, dyslipemia, diabetes mellitus, smoking habit, obesity, $\mathrm{p}<0.01$ for all) and other comorbidities such as chronic renal failure, any lung disease, cerebrovascular disease and oncologic antecedent ( $\mathrm{p}<0.01$, for all).

Regarding previous treatments, signs and symptoms, Table 1 displays the main findings, compared with patients without heart disease.

In addition, Table 2 presents the main analytic findings at the time of admission and in hospital management strategies. Chest X-ray exhibited any acute lung abnormality in more than $70 \%$, mostly bilateral $(57.6 \%)$. In this setting, heart patients needed more respiratory and circulatory support and presented higher in hospital events (Table 3 ). The specific drug most frequently used was hydroxychloroquine (72\%), followed by antibiotics and any antiviral drug (mostly lopinavir/ritonavir). Nevertheless, the heart disease group received a different pattern of treatment, characterized by more systemic corticoids, antibiotics, but less hydroxychloroquine, antivirals or tocilizumab (Table 2).

\section{Influence of a previous heart condition}

Assessing the whole sample epidemiologic profile, gender (male), age (increasing) and the presence of hypertension, dyslipemia, diabetes mellitus, obesity (body mass index $>30$ ), renal insufficiency, any lung disease, any heart disease, previous cerebrovascular condition, connective or liver disease, any cancer or immunosuppressive condition displayed a significantly higher mortality $(\mathrm{p}<0.01$ in all) in the univariate analysis.

Considering these variables in the multivariate assessment (Table 4), the following factors were considered independent risk factors: age, hypertension, chronic renal failure, any cancer and any heart disease (hazard ratio [HR] 1.62; 95\% confidence interval [CI] 1.29-2.03).

\section{Outcomes inside the heart disease cohort}

Focusing on those with an underlying heart condition, these patients presented higher mortality $(39.7 \%$ vs. $15.5 \%$, non-adjusted odds ratio [OR] 3.58; 95\% CI 2.95-4.34; $\mathrm{p}<0.001$; Figs. 2, 3).
Heart patients, also, suffered more frequent inhospital events, such as heart failure, acute renal failure, respiratory insufficiency, sepsis, systemic infammatory response syndrome and clinically relevant bleedings, (all, $\mathrm{p}<0.001$ ). Table 3 discusses this further and depicts the raw in-hospital events regarding the type of relevant heart condition group.

In the multivariate analysis for mortality, considering only the heart disease group, the following were included in the final model: age 70 years or more, hypertension, diabetes mellitus, chronic renal failure, use of oral anticoagulants, Vitamin D supplements, myalgia/arthralgia, $\mathrm{O}_{2}$ saturation $<92 \%$, decreased blood pressure, elevated D dimer, elevated $\mathrm{C}$ reactive protein, elevated lactate dehydrogenase (LDH), invasive mechanical ventilation, prone during admission, use of corticoids, hydroxychloroquine and angiotensin converting enzyme inhibitors/angiotensin receptor blockers (ACEIs/ARBs) during admission. Tachypnea and use of high flow nasal cannulas were excluded for potential collinearity with other variables $\left(\mathrm{O}_{2}\right.$ saturation and mechanical ventilation). Of those, age (HR 4.3; 95\% CI 2.23-8.28), hypertension (HR 1.7; 95\% CI 1.01-2.89), $\mathrm{O}_{2}$ saturation < 92\% (HR 3.59; 95\% CI 2.43-5.31), an elevated LDH (HR 1.66; 95\% CI 1.01-2.73), the use of mechanical ventilation (HR 2.11; 95\% CI 1.17-3.80) remained in the model as risk factors while the use of in hospital ACEIs/ARBS (HR 0.34; 95\% CI 0.20-0.49) and hydroxychloroquine (HR 0.69; 95\% CI 0.45-0.99) resulted as potential protective factors.

\section{Discussion}

The present study is a cohort study among patients with COVID-19 after discharge, reviewing the direct impact on mortality of previous heart diseases.

Regarding COVID-19, any kind of heart disease is probably a truly relevant condition. First, because compared to the general population, the incidence of cardio-cerebrovascular disease in patients with COVID-19 is much higher [14]. Second, because patients with hypertension, cardio-cerebrovascular diseases or diabetes are more likely to develop into severe/intensive care unit (ICU) cases or die after SARS-CoV-2 infection $[5,7,14]$. The overall proportion of hypertension, cardio-cerebrovascular problems and diabetes were about two-fold, three-fold and two-fold, respectively, higher in ICU/severe cases than in their non-ICU/severe counterparts [14]. In the present cohort, it was observed that outcomes of patients 
Table 1. Clinical features, previous treatments and presentation symptoms before admission overall and stratified among patients with heart disease or without heart disease.

\begin{tabular}{|c|c|c|c|c|}
\hline \multirow[b]{4}{*}{ Age [years] } & \multirow{4}{*}{$\begin{array}{c}\text { All patients* } \\
N=2798 \\
\text { No./total no. (\%) } \\
/ 2788\end{array}$} & \multicolumn{2}{|c|}{ Heart disease** } & \multirow[t]{3}{*}{$\mathbf{P}$} \\
\hline & & Absent $(N=2116)$ & Present $(\mathrm{N}=682)$ & \\
\hline & & \multicolumn{2}{|c|}{ No./total no. $(\%)$} & \\
\hline & & $/ 2109$ & $/ 679$ & $<0.001$ \\
\hline Median (IQR) [years] & $67(53.0-78.0)$ & $63(49-74)$ & $75(70-85)$ & \\
\hline \multicolumn{5}{|l|}{ Distribution [years]: } \\
\hline $0-14$ & $24(0.9)$ & $22(1.0)$ & $2(0.3)$ & \\
\hline $15-49$ & $540(19.4)$ & $514(24.4)$ & $26(3.8)$ & \\
\hline $50-64$ & $674(24.2)$ & $592(28.1)$ & $83(12.2)$ & \\
\hline$\geq 65$ & $1544(55.5)$ & $981(46.5)$ & $568(83.7)$ & \\
\hline Gender: & /2798 & & & 0.001 \\
\hline Female & 1111 (39.7) & $878(41.5)$ & $233(34.2)$ & \\
\hline Male & $1687(59.5)$ & $1238(58.5)$ & $449(65.8)$ & \\
\hline Race: & /2798 & & & $<0.001$ \\
\hline Caucasian & $2351(84.0)$ & $1743(82.4)$ & $608(89.1)$ & \\
\hline Latin & $357(12.8)$ & $301(14.2)$ & $56(8.2)$ & \\
\hline Asian & $34(1.2)$ & $32(1.5)$ & $2(.3)$ & \\
\hline Black & $32(1.1)$ & $21(1.0)$ & $11(1.6)$ & \\
\hline Other & $24(0.9)$ & $19(0.9)$ & $45(0.3)$ & \\
\hline Hypertension & /2784 & /2106 & /678 & $<0.001$ \\
\hline Yes & $1370(49.2)$ & $1265(60.1)$ & $149(22.0)$ & \\
\hline Dyslipidemia & $/ 2767$ & /2099 & /668 & $<0.001$ \\
\hline Yes & $971(35.1)$ & $602(28.7)$ & $369(55.2)$ & \\
\hline Diabetes mellitus ( 1 or 2 ) & /2677 & /2039 & /638 & $<0.001$ \\
\hline Yes & $482(18.0)$ & $292(14.3)$ & $190(29.8)$ & \\
\hline Obesity (BMI > $\left.30 \mathrm{~kg} / \mathrm{m}^{2}\right)$ & /2023 & $/ 1527$ & 1496 & 0.006 \\
\hline Yes & $459(22.7)$ & $324(21.2)$ & $135(27.2)$ & \\
\hline CRI & /2681 & /2045 & /636 & $<0.001$ \\
\hline Yes & $192(7.2)$ & $97(4.7)$ & 95 (14.9) & \\
\hline Smoking habit: & /2494 & /1887 & $/ 607$ & 0.147 \\
\hline No & $2321(93.1)$ & 1764 (93.5) & $557(91.8)$ & \\
\hline Current & $173(6.9)$ & $123(6.5)$ & $50(8.2)$ & \\
\hline Lung disease: & & & & $<0.001$ \\
\hline No & $2266(81.0)$ & $171(83.7)$ & $495(72.6)$ & \\
\hline Asma & $146(5.2)$ & $121(5.7)$ & $25(3.7)$ & \\
\hline COPD & $197(7.0)$ & $108(5.1)$ & $89(13.0)$ & \\
\hline Interstitial & $19(0.7)$ & $9(0.4)$ & $10(1.5)$ & \\
\hline Restrictive & $23(0.8)$ & $13(0.6)$ & $10(1.5)$ & \\
\hline Other & $147(5.3)$ & $94(4.4)$ & $53(7.8)$ & \\
\hline Any cancer & $/ 2710$ & $/ 2056$ & $/ 654$ & $<0.001$ \\
\hline Yes & $367(13.5)$ & $240(11.7)$ & $127(19.4)$ & \\
\hline Any immunosuppressive disease & $/ 2491$ & /1888 & /603 & 0.060 \\
\hline Yes & $195(7.8)$ & $137(7.3)$ & $58(9.6)$ & \\
\hline
\end{tabular}


Table 1 (cont.). Clinical features, previous treatments and presentation symptoms before admission overall and stratified among patients with heart disease or without heart disease.

\begin{tabular}{|c|c|c|c|c|}
\hline & \multirow{3}{*}{$\begin{array}{c}\text { All patients* } \\
\text { N }=2798 \\
\text { No./total no. (\%) }\end{array}$} & \multicolumn{2}{|c|}{ Heart disease** } & \multirow[t]{3}{*}{$\mathbf{P}$} \\
\hline & & Absent ( $N=2116)$ & Present $(N=682)$ & \\
\hline & & \multicolumn{2}{|c|}{ No./total no. $(\%)$} & \\
\hline Dependency level: & & & & $<0.001$ \\
\hline Not disclosed & $53(1.9)$ & $38(1.8)$ & $15(2.2)$ & \\
\hline None & 2397 (85.7) & 1903 (89.9) & $494(72.4)$ & \\
\hline Partially & $249(8.9)$ & $115(5.4)$ & $134(19.6)$ & \\
\hline Totally & $99(3.5)$ & $60(2.8)$ & $39(5.7)$ & \\
\hline Home oxygen therapy & $/ 2762$ & $/ 2089$ & $/ 673$ & $<0.001$ \\
\hline Yes & $93(3.4)$ & $50(2.4)$ & $43(6.4)$ & \\
\hline ASA & $/ 2747$ & /2076 & /671 & $<0.001$ \\
\hline Yes & $429(15.6)$ & $191(9.2)$ & $238(35.5)$ & \\
\hline Oral anticoagulation & /2732 & /2064 & /668 & $<0.001$ \\
\hline Yes & $322(11.8)$ & $33(1.6)$ & $289(43.3)$ & \\
\hline ACEls/ARBs & $/ 2759$ & /2092 & /667 & $<0.001$ \\
\hline Yes & $979(35.5)$ & $596(28.5)$ & $383(57.4)$ & \\
\hline Beta-blockers & $/ 2740$ & /2067 & /673 & $<0.001$ \\
\hline Yes & $483(17.7)$ & $129(6.2)$ & $354(52.6)$ & \\
\hline Inhaled beta agonist & 2737 & /2080 & /657 & $<0.001$ \\
\hline Yes & $289(10.6)$ & $169(8.1)$ & $120(18.3)$ & \\
\hline Inhaled corticoids & $/ 2743$ & $/ 2078$ & $/ 665$ & $<0.001$ \\
\hline Yes & $241(8.8)$ & $150(7.2)$ & $91(13.7)$ & \\
\hline Vitamin D supplements & /2718 & /2067 & $/ 651$ & $<0.001$ \\
\hline Yes & $287(10.6)$ & $165(8.0)$ & $122(18.7)$ & \\
\hline Tachypnea (> 22 bpm) & $/ 2640$ & $/ 2001$ & $/ 639$ & $<0.001$ \\
\hline Yes & $666(25.2)$ & $443(22.1)$ & $223(34.9)$ & \\
\hline Hypo-anosmia & $/ 2510$ & /1892 & /618 & 0.061 \\
\hline Yes & $176(7.0)$ & $143(7.6)$ & $33(5.3)$ & \\
\hline Dysgeusia & $/ 2507$ & $/ 1889$ & $/ 618$ & 0.180 \\
\hline Yes & $198(7.9)$ & $157(8.3)$ & $41(6.6)$ & \\
\hline Sore throat & $/ 2728$ & $/ 260$ & /399 & 0.005 \\
\hline Yes & $1889(69.2)$ & $158(60.7)$ & $257(64.4)$ & \\
\hline Fever & $/ 2754$ & $/ 2085$ & $/ 669$ & $<0.001$ \\
\hline Yes & $2235(81.2)$ & $1735(83.2)$ & $500(74.7)$ & \\
\hline Cough & $/ 2734$ & /2073 & $/ 661$ & $<0.001$ \\
\hline Yes & $1893(69.2)$ & $1477(71.2)$ & $416(62.9)$ & \\
\hline Diarrhea & $/ 2632$ & /1992 & $/ 640$ & 0.004 \\
\hline Yes & $510(19.4)$ & $411(20.6)$ & 99 (15.5) & \\
\hline Myalgia/arthralgia & $/ 2651$ & /2009 & $/ 642$ & $<0.001$ \\
\hline Yes & $884(33.3)$ & $713(35.5)$ & $171(26.6)$ & \\
\hline $\mathrm{O}_{2}$ saturation $<92 \%$ & $/ 2699$ & /2043 & $/ 656$ & $<0.001$ \\
\hline Yes & $893(33.1)$ & $572(28.0)$ & $321(48.9)$ & \\
\hline Abnormal BP (SBP $<90 /<60 \mathrm{mmHg}$ ) & $/ 2758$ & $/ 2091$ & $/ 667$ & $<0.001$ \\
\hline Yes & $109(5.2)$ & $109(5.2)$ & $81(12.1)$ & \\
\hline
\end{tabular}

* Some data are missing at the time of interim analysis. Calculations and percentages are expressed upon the recorded data as are displayed in the table (recorded/total available).

**Comparisons and $\mathrm{p}$ values are applied to heart disease absence or presence.

ACEI/ARB - angiotensin converting enzyme inhibitors/angiotensin receptors blockers; ASA — acelylsalicylic acid; BMI — body mass index; $\mathrm{BP}$ - blood pressure; CRI — chronic renal insufficiency; COPD — chronic obstructive pulmonary disease; SBP - systolic blood pressure 
Table 2. Relevant analytical results (early at admission) and in hospital management regarding the presence of heart disease or no presence of heart disease.

\begin{tabular}{|c|c|c|c|c|}
\hline & \multirow{2}{*}{$\begin{array}{l}\text { All patients* } \\
N=2798\end{array}$} & \multicolumn{2}{|c|}{ Heart disease** } & \multirow[t]{2}{*}{$\mathbf{P}$} \\
\hline & & Absent $(N=2116)$ & Present $(\mathrm{N}=682)$ & \\
\hline Elevated $\mathrm{D}$ dimer & $/ 2394$ & $/ 1825$ & $/ 569$ & 0.001 \\
\hline Yes & $1538(64.2)$ & $1140(62.5)$ & $398(69.9)$ & \\
\hline Elevated procalcitonin & $/ 2146$ & $/ 1631$ & $/ 515$ & 0.004 \\
\hline Yes & $527(24.6)$ & $376(23.1)$ & $151(29.3)$ & \\
\hline Elevated $\mathrm{C}$ reactive protein & $/ 2724$ & $/ 2059$ & /665 & $<0.001$ \\
\hline Yes & $2456(90.2)$ & $1831(88.9)$ & $625(94.0)$ & \\
\hline Elevated troponin & $/ 1325$ & /969 & /356 & $<0.001$ \\
\hline Yes & $222(16.8)$ & $119(12.3)$ & $103(28.9)$ & \\
\hline Elevated LDH & $/ 2503$ & $/ 1889$ & $/ 614$ & 0.014 \\
\hline Yes & $1820(72.7)$ & 1350 (71.5) & $470(76.5)$ & \\
\hline Elevated creatinine (> $1.5 \mathrm{mg} / \mathrm{dL}$ ) & $/ 2319$ & /1764 & $/ 555$ & $<0.001$ \\
\hline Yes & $375(16.2)$ & $223(12.6)$ & $152(27.4)$ & \\
\hline White count cell $(\leq 4000 / \mu \mathrm{L})$ & /2709 & /2056 & $/ 653$ & 0.688 \\
\hline Yes & $462(17.1)$ & $354(17.2)$ & $108(16.5)$ & \\
\hline Lymphocytes count $(\leq 1500 / \mu \mathrm{L})$ & $/ 2625$ & /2000 & $/ 625$ & $<0.001$ \\
\hline Yes & $1980(75.4)$ & $1474(73.7)$ & $506(81.0)$ & \\
\hline Hemoglobin levels ( $\leq 12 \mathrm{~g} / \mathrm{dL}$ ) & /2695 & /2047 & /648 & $<0.001$ \\
\hline Yes & $681(25.3)$ & $427(20.9)$ & $254(39.2)$ & \\
\hline Platelet counts $(\leq 150,000 / \mu \mathrm{L})$ & /2701 & $/ 2053$ & /648 & $<0.001$ \\
\hline Yes & $728(27.0)$ & $483(23.5)$ & $245(37.8)$ & \\
\hline \multicolumn{5}{|l|}{ MANAGEMENT } \\
\hline High flow nasal cannula & /2686 & /2026 & $/ 660$ & $<0.001$ \\
\hline Yes & $492(18.3)$ & $325(16.0)$ & $167(25.3)$ & \\
\hline Noninvasive mechanical ventilation & /2684 & /2026 & /658 & 0.027 \\
\hline Yes & $390(14.5)$ & $277(13.7)$ & $113(17.2)$ & \\
\hline Invasive mechanical ventilation & /2646 & /2008 & /638 & 0.927 \\
\hline Yes & $168(6.3)$ & $127(6.3)$ & $41(6.4)$ & \\
\hline Prone during admission & /2665 & /2018 & $/ 647$ & 0.461 \\
\hline Yes & $246(9.2)$ & $191(9.5)$ & $55(8.5)$ & \\
\hline Circulatory/ECMO support & /948 & $/ 754$ & /194 & 0.822 \\
\hline Yes & $4(0.4)$ & $3(0.4)$ & $1(0.5)$ & \\
\hline Use of corticoids & $/ 2693$ & /2039 & $/ 654$ & $<0.001$ \\
\hline Yes & $604(22.4)$ & $415(20.4)$ & $189(28.9)$ & \\
\hline Use of hydroxichloroquine & $/ 2728$ & /2067 & $/ 661$ & 0.001 \\
\hline Yes & $2306(84.5)$ & $1775(85.9)$ & $531(80.3)$ & \\
\hline Use of antivirals & $/ 2726$ & /2066 & $/ 660$ & $<0.001$ \\
\hline Yes & $1795(65.8)$ & $1415(68.5)$ & $380(57.6)$ & \\
\hline Use of tocilizumab & $/ 2681$ & /2036 & $/ 645$ & 0.012 \\
\hline Yes & $183(6.8)$ & $153(7.5)$ & $30(4.7)$ & \\
\hline Use of antibiotics & $/ 2625$ & /1993 & /632 & 0.013 \\
\hline Yes & $1953(74.4)$ & $1459(73.2)$ & $494(78.2)$ & \\
\hline Use of ACEIs/ARBs during stay & $/ 2598$ & /1981 & $/ 617$ & $<0.001$ \\
\hline Yes & $464(17.9)$ & $291(14.7)$ & $173(28.0)$ & \\
\hline
\end{tabular}

*Some data are missing at the time of interim analysis. Calculations and percentages are expressed upon the recorded data as are displayed in the table (recorded/total available).

**Comparisons and $\mathrm{p}$ values are applied to heart disease absence or presence.

ACEI/ARB - angiotensin converting enzyme inhibitors/angiotensin receptors blockers; ECMO - extracorporeal membrane oxygenation;

$\mathrm{LDH}$ - lactate dehydrogenase 


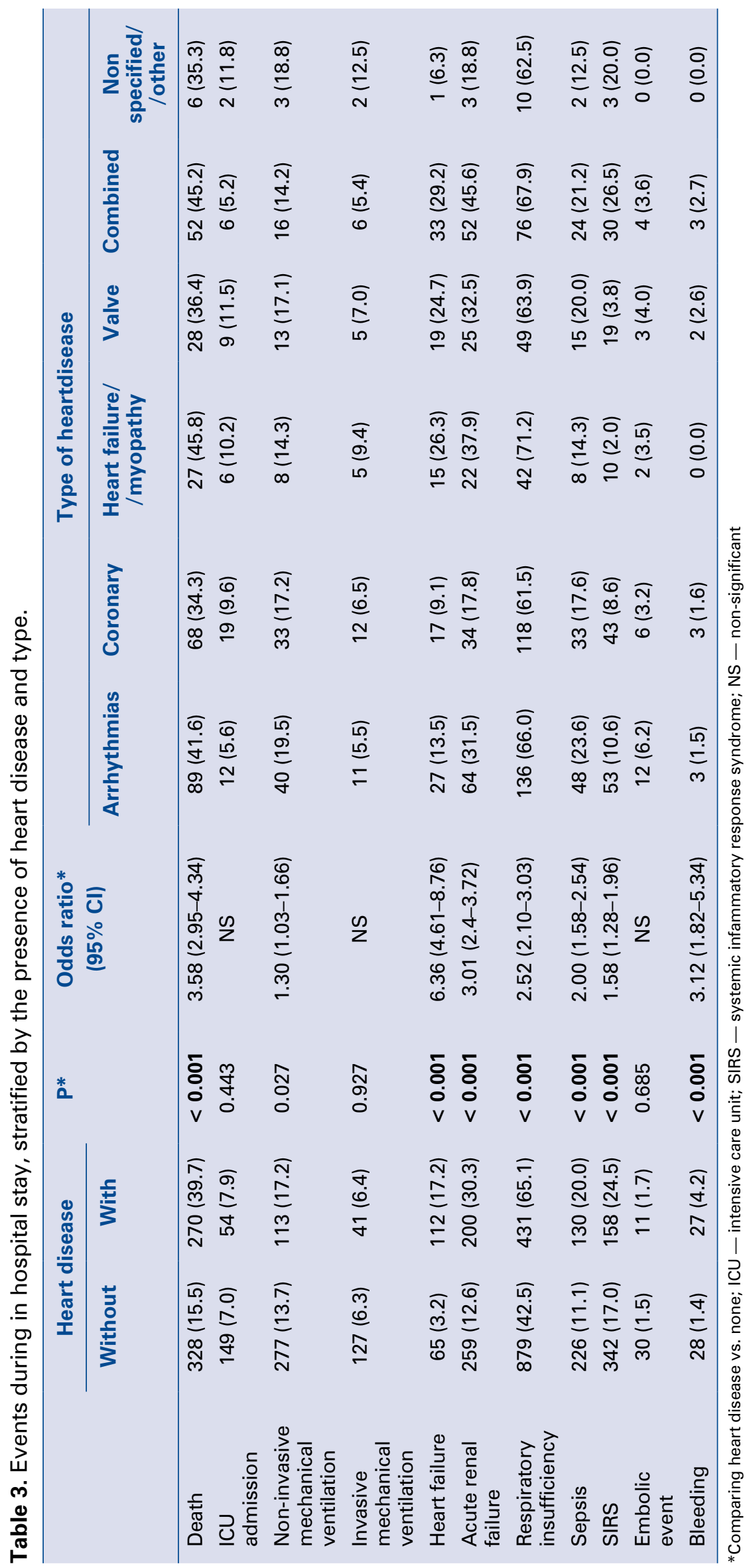


Table 4. Variables remaining in the model regarding mortality (multivariate analysis by Cox regression; backward: Wald)*.

\begin{tabular}{lccc}
\hline Characteristic & Hazard ratio & 95\% confidence interval & $\mathbf{P}$ \\
\hline Age 70 years or more & 4.05 & $3.02-5.42$ & $<\mathbf{0 . 0 0 1}$ \\
Hypertension & 1.39 & $1.07-1.81$ & $\mathbf{0 . 0 1 3}$ \\
Any heart condition & 1.62 & $1.29-2.03$ & $<\mathbf{0 . 0 0 1}$ \\
Chronic renal failure & 1.80 & $1.38-2.37$ & $<\mathbf{0 . 0 0 1}$ \\
Any cancer & 1.36 & $1.06-1.76$ & $\mathbf{0 . 0 1 6}$ \\
Any cerebrovascular condition & 1.30 & $0.98-1.74$ & 0.070 \\
\hline
\end{tabular}

Variables included in the clinical model*: For modeling purposes, at this point only age, gender and relevant comorbidities were considered. Thus, those variables with $\mathrm{p}$ values $<0.05$ regarding mortality were included in the multivariate analysis. The variables accepted were: age 70 years or more, gender, race, hypertension, dyslipemia, diabetes mellitus, obesity, chronic renal failure, any lung disease, cerebrovascular conditions, any heart disease, connective disease, any cancer, immunosuppressive condition, and any liver disease. The reference value was the absence of the precise condition. Previous medications were excluded for potential collinearity with the other mentioned variables (comorbidities)

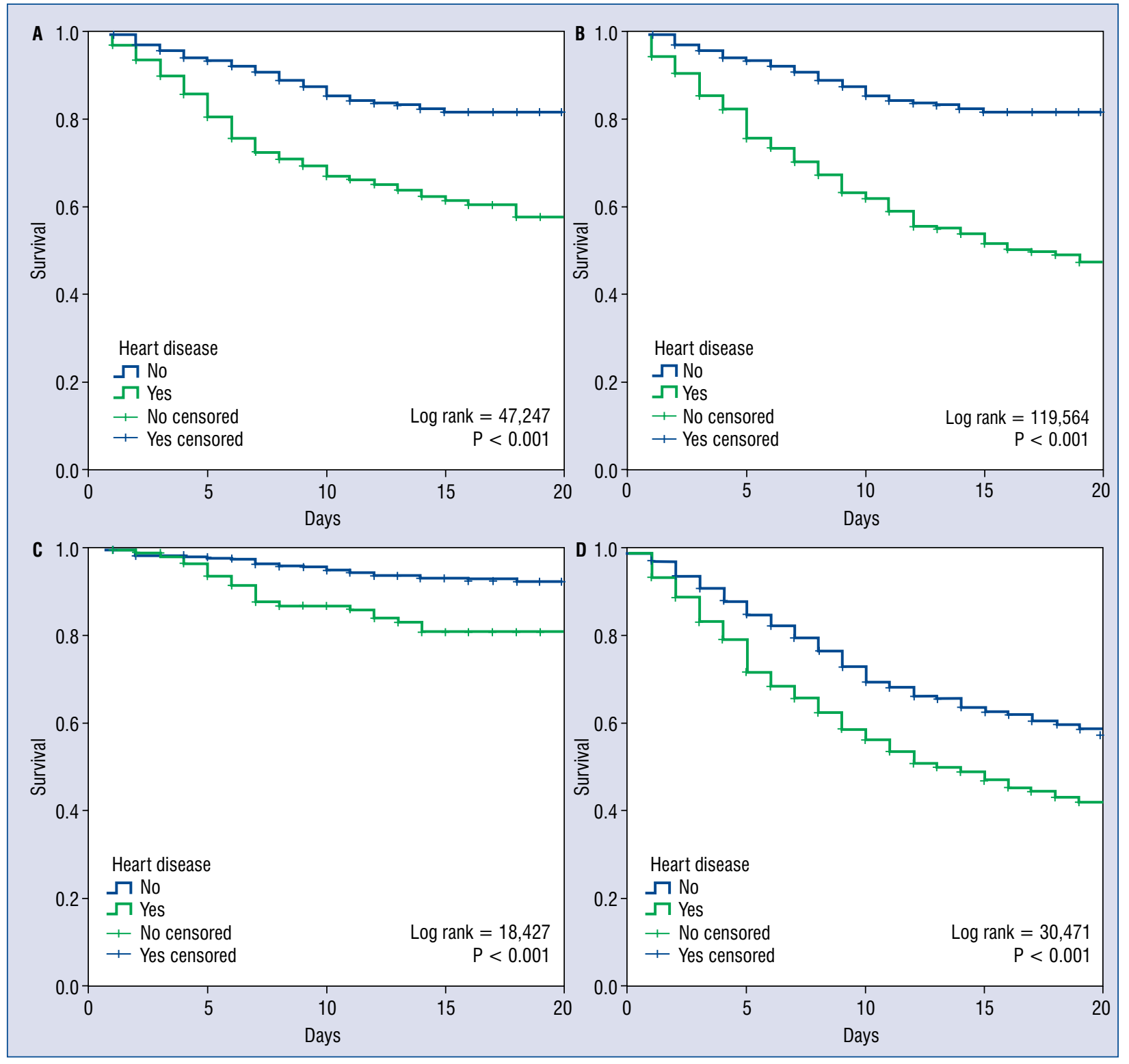

Figure 2. The Kaplan-Meier survival analysis stratifying for gender and age; $T_{0}=$ admission date; Considering only females (A) or males (B), less than 70 years old (C) and $\geq 70$ years old (D). 


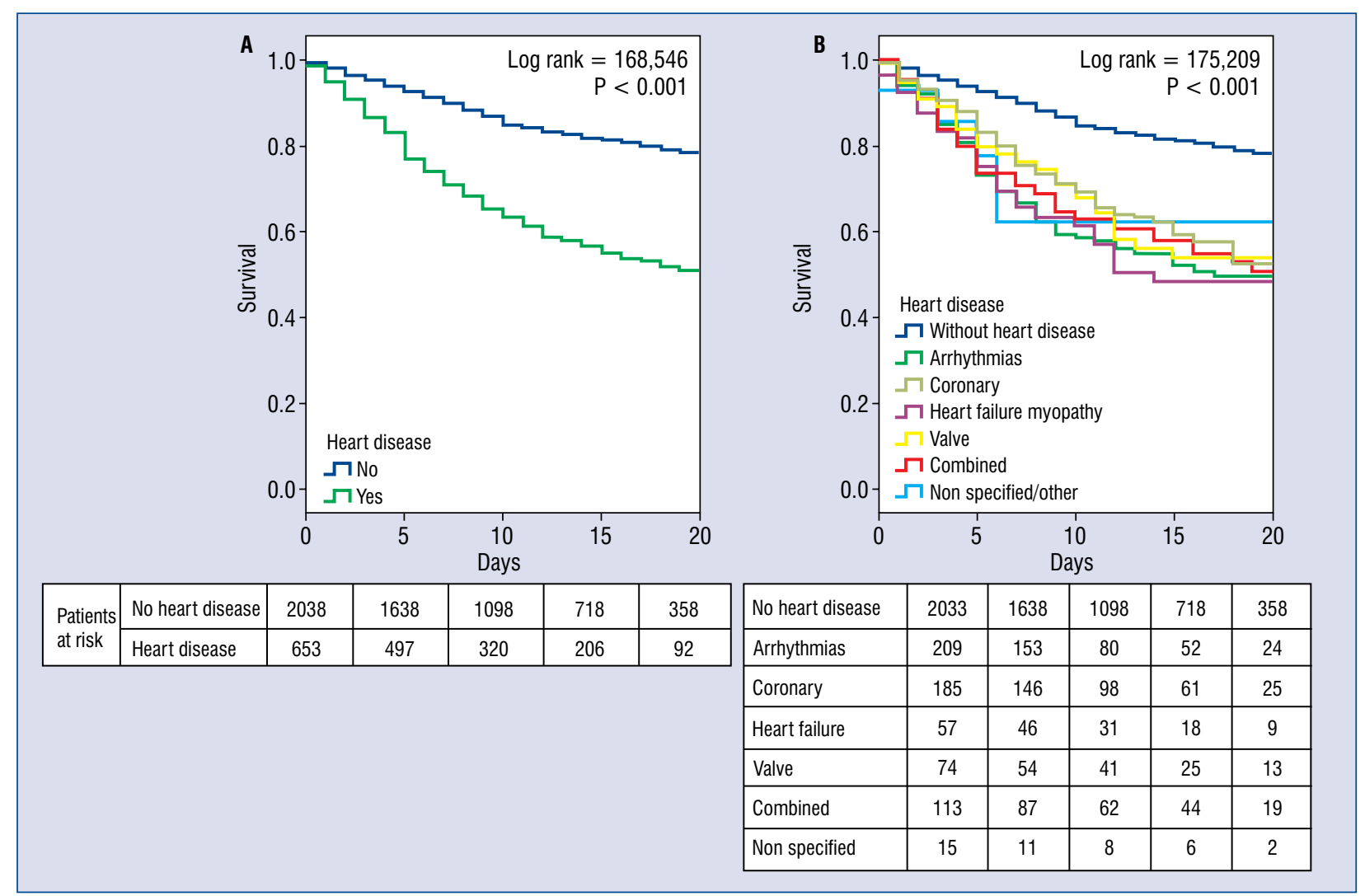

Figure 3. Kaplan-Meier survival landmark analysis; $\mathrm{T}_{0}=$ admission date; $\mathbf{A}$. Assessing no heart disease vs. any type of heart condition; B. Same comparison but regarding type of heart diseases.

with any heart disease were clearly worse regarding mortality and other in-hospital complications. Overall, these findings suggest that preexisting heart problems marked a frailty point for COVID-19 patients warranting close surveillance, intensive management and were considered low threshold for admission.

On the other hand, at least $8.0 \%$ of COVID-19 patients suffered any kind of acute cardiac injury, but further analysis pointed out that the incidence of myocardial injury is much higher in ICU/severe patients, about 13 -fold more than non-ICU/cardiac patients $[9,14]$.

Obviously, part of this frailty can be explained because of a different clinical profile (elderly, more cardiovascular risk factors, renal disease and other COVID-19 factors) among heart and no-heart disease cohort, but not entirely. Altogether, heart disease seems to be primarily a risk factor for bad prognosis in COVID-19 [15]. This way, usually cardiovascular involvement measured by troponin levels and cardiovascular complications are higher in heart disease patients, as we observed in HOPE.
This is expected because it has been reported that the COVID-19, which supposes a severe global aggression, could primarily involve the heart and cardiovascular systems. Several mechanisms are at play in this regard, either by direct or indirect mechanisms, in adults but also in infants $[11,15]$. Anyway, elevated cardiac troponin seems to point toward a worse prognosis [16].

The viral (SARS-CoV-2) infection is prompted by the binding of the virus' spike protein to angiotensinconverting enzyme 2 (ACE2) [16]. The expression of this ACE2 in the heart has been described to be lower than that in other organs, such as the intestine and kidney, but higher than in the lung which serves as a main target organ of the virus, indicating a potential infection susceptibility of the human heart [17, 18]. In some specific circumstances, this heart susceptibility could be theoretically higher, since ACE2 expression has been reported to be significantly increased in patients with heart failure, post myocardial infarction and diabetes [18-20].

Some of the proposed pathophysiological mechanisms would be: 
- Direct heart damage. Viral infection directly causes damage to cardiomyocyte. According to Oudit et al. [21], SARS-CoV viral RNA was detected in $35 \%$ of autopsied human heart samples from SARS-CoV infected patients during the past Toronto SARS outbreak. Of note, SARS-CoV and SARS-CoV-2 present high structural similarity between their receptor-binding domains [22]. Additionally, virus-infected cells can be injured, subsequently disturbing the micro-environment of the myocardium. SARS-CoV-2 infection in the human heart might attack pericytes as well, produce endothelial shedding and cause capillary dysfunction and induce micro-circulation disorders $[15,18]$. In our series, the specific type of heart disease with higher mortality with frequent in-hospital complications was heart failure/myopathy suggesting that structurally weaker hearts could pose higher frailty.

- Hypoxia-induced myocardial injury. Because of lung pathology, pneumonia, respiratory distress syndrome, or the previously mentioned macro or micro vessel direct toxicity [15]. This condition, decreases the cell energy supply, leading to anaerobic fermentation, producing intracellular acidosis and oxygen free radicals to dismantle the phospholipid layer of the cell membrane. Moreover, hypoxia-induced influx of calcium also primes to injury and apoptosis of cardiac cells [15].

- Production of procoagulant factors predisposing to thrombosis, similar to the increase of myocardial infarctions reported after influenza infection [15, 23]. In fact, abnormal coagulation parameters and disseminated intravascular coagulation has been noted in COVID-19 [15] potentially contributing to damage the myocardium through thrombosis or ischemic events.

- Local inflammation. Although there are early reports of myocarditis [24], even fulminant, the exact mechanism is not clear, since lymphocyte infiltrates were not found in COVID-19 patients' autopsy $[15,25]$.

- Probably, a significant depletion and dysregulation of $T$ cells can probably contribute to the cytokine storm (increased IL-2, IL-6, IL-10, GCSF, IFN- $\gamma$, MCP-1 and TNF- $\alpha$ ) leading to the multiorgan damage setting depicted in COVID-19. Cardiac damage by this deleterious condition could be analogous to that reported in CAR-T (chimeric antigen receptor $\mathrm{T}$ cell therapies used in relapsing hematological malignancies).
Finally, last but not least, many specific drugs used for COVID-19 can cause cardiac side effects, arrhythmias or other cardiovascular disorders (hydroxychloroquine, antivirals, antibiotics, some immunomodulators). Different drug patterns were found when comparing patients with and without heart conditions. Therefore, during treatment of this condition, especially with the use of certain drugs, the risk of cardiac toxicity must be closely monitored, but to avoid depriving heart patients of potentially beneficial treatments. On the other side, special attention should be given to cardiovascular protective measures during management of COVID-19, since those patients have high risk of complications [13, 25-27]. In this aspect, the crucial role of ACEIs/ARBs needs to be taken into account $[9,10,28,29]$. Despite under scientific review, preliminary data seem to warrant its maintenance in patients already on these meds at admission. Additionally, the present findings display a potential mortality benefit when maintaining these treatments in this setting (OR 0.34; 95\% CI $0.20-0.49 ; \mathrm{p}<0.001$; Table 4$)$.

\section{Limitations of the study}

The main limitation is set by the study observational design and selecting only cases with higher risk profile or severe forms needing hospital admission. In addition, the definition of the variables, the specific type and degree of heart disease and the reporting for the events could present certain grade of variation among centers, countries and the precise moment in their pandemic curve. However, this would probably reflect the variation that medical practice has in real life. About the treatment applied, at all times it was decided by the attending physician. While these observations give us an overall idea of the treatment of the disease in this precise cohort, they do not produce information as robust as a clinical trial would do [30].

Thus, the only aim was to generate hypotheses; nevertheless, HOPE's present analysis probably reveals a pragmatic depiction of the outcomes and prognosis of patients with prevalent heart conditions who are admitted with COVID-19, a challenge for modern medicine [30, 31].

\section{Conclusions}

An underlying heart disease is an adverse prognostic factor for patients suffering COVID-19. Its presence could be related with varying clinical drug management and could benefit from main- 
taining treatment with ACEIs or ARBs during in-hospital stay.

\section{Acknowledgements}

Cardiovascular Excellence SL, for their essential support in the database and HOPE webpage. All HOPE researchers.

\section{Funding}

Non-conditioned grant (Fundación Interhospitalaria para la Investigación cardiovascular, FIC. Madrid, Spain). This nonprofit institution had no role in the study design; collection, analysis, interpretation of data; in the writing of the report; nor in the decision to submit the paper for publication

\section{HOPE COVID-19 Investigators, Scientific Committee And Collaborators: see Supplemen- tary Appendix}

\section{Conflict of interest: None declared}

\section{References}

1. WHO. WHO statement regarding cluster of pneumonia cases in Wuhan, China. https://www.who.int/china/news/detail/0901-2020-who-statement-regarding-cluster-of-pneumonia-casesin-wuhan-china (Accesed 3rd May 2020).

2. WHO. http://www.euro.who.int/en/health-topics/health-emergencies/coronavirus-covid-19/news/news/2020/3/who-announces-covid-19-outbreak-a-pandemic (Accesed 3rd May 2020).

3. Dong E, Du H, Gardner L. An interactive web-based dashboard to track COVID-19 in real time. Lancet Infect Dis. 2020; 20(5): 533-534, doi: 10.1016/S1473-3099(20)30120-1, indexed in Pubmed: 32087114.

4. Hulot JS. COVID-19 in patients with cardiovascular diseases. Arch Cardiovasc Dis. 2020; 113(4): 225-226, doi: 10.1016/j. acvd.2020.03.009, indexed in Pubmed: 32245656.

5. Shi S, Qin Mu, Shen Bo, et al. Association of cardiac injury with mortality in hospitalized patients with COVID-19 in Wuhan, China. JAMA Cardiol. 2020; 5(7): 802-810, doi: 10.1001/jamacardio.2020.0950, indexed in Pubmed: 32211816.

6. Centers for Disease Control and Prevention. Coronavirus disease 2019 (COVID19). https://www.cdc.gov/coronavirus/2019ncov/ /needextraprecautions/peopleathigherrisk.html?CDC_AA_ refVal $=$ https $\% 3 \mathrm{~A} \% 2 \mathrm{~F} \% 2 \mathrm{Fw} w \mathrm{w} . c d c$.gov $\% 2 \mathrm{Fcoronavirus}$ \%2F2019-ncov\%2Fspecific-groups\% 2Fhigh-riskcomplications. html (Accessed 22 April 2020).

7. Madjid M, Solomon S, Vardeny O. Cardiac Implications of Novel Coronavirus (COVID-19). In: Committee ASaQ, editor. ACC Clinical Bulletin. USA: ACC; 2020.

8. Wang $\mathrm{D}, \mathrm{Hu} \mathrm{Bo}, \mathrm{Hu} \mathrm{C}$, et al. Clinical characteristics of 138 hospitalized patients with 2019 novel coronavirus-infected pneumonia in Wuhan, China. JAMA. 2020; 323(11): 1061-1069, doi: 10.1001/ jama.2020.1585, indexed in Pubmed: 32031570.

9. Zheng YY, Ma YT, Zhang JY, et al. COVID-19 and the cardiovascular system. Nat Rev Cardiol. 2020; 17(5): 259-260, doi: 10.1038/s41569-020-0360-5, indexed in Pubmed: 32139904.
10. Bansal M. Cardiovascular disease and COVID-19. Diabetes Metab Syndr. 2020; 14(3): 247-250, doi: 10.1016/j.dsx.2020.03.013, indexed in Pubmed: 32247212.

11. Aghagoli G, Gallo Marin B, Soliman LB, et al. Cardiac involvement in COVID-19 patients: Risk factors, predictors, and complications: A review. J Card Surg. 2020; 35(6): 1302-1305, doi: 10.1111/jocs.14538, indexed in Pubmed: 32306491.

12. Holy EW, Jakob P, Manka R, et al. Impact of a nationwide COVID-19 lockdown on acute coronary syndrome referrals. Cardiol J. 2020; 27(5): 633-635, doi: 10.5603/CJ.a2020.0091, indexed in Pubmed: 32643140.

13. Núñez-Gil IJ, Estrada V, Fernández-Pérez C, et al. Health Outcome Predictive Evaluation for COVID 19 international registry (HOPE COVID-19), rationale and design. Contemp Clin Trials Commun. 2020; 20: 100654, doi: 10.1016/j.conctc.2020.100654, indexed in Pubmed: 32989425.

14. Li Bo, Yang J, Zhao F, et al. Prevalence and impact of cardiovascular metabolic diseases on COVID-19 in China. Clin Res Cardiol. 2020; 109(5): 531-538, doi: 10.1007/s00392-020-01626-9, indexed in Pubmed: 32161990.

15. Zhu H, Rhee JW, Cheng P, et al. Correction to: cardiovascular complications in patients with COVID-19: consequences of viral toxicities and host immune response. Curr Cardiol Rep. 2020; 22(5): 36, doi: 10.1007/s11886-020-01302-4, indexed in Pubmed: 32405913.

16. Lorente-Ros A, Monteagudo Ruiz JM, Rincón LM, et al. Myocardial injury determination improves risk stratification and predicts mortality in COVID-19 patients. Cardiol J. 2020; 27(5): 489-496, doi: 10.5603/CJ.a2020.0089, indexed in Pubmed: 32589258.

17. Hoffmann M, Kleine-Weber H, Schroeder S, et al. SARS-CoV-2 Cell Entry Depends on ACE2 and TMPRSS2 and Is Blocked by a Clinically Proven Protease Inhibitor. Cell. 2020; 181(2): 271-280.e8, doi: 10.1016/j.cell.2020.02.052, indexed in Pubmed: 32142651.

18. Chen $\mathrm{Li}$, Hao G. The role of angiotensin-converting enzyme 2 in coronaviruses/influenza viruses and cardiovascular disease. Cardiovasc Res. 2020; 116(12): 1932-1936, doi: 10.1093/cvr/ cvaa093, indexed in Pubmed: 32267499.

19. Burrell LM, Risvanis J, Kubota E, et al. Myocardial infarction increases ACE2 expression in rat and humans. Eur Heart J. 2005; 26(4): 369-75; discussion 322, doi: 10.1093/eurheartj/ehi114, indexed in Pubmed: 15671045.

20. Soro-Paavonen A, Gordin D, Forsblom C, et al. FinnDiane Study Group. Circulating ACE2 activity is increased in patients with type 1 diabetes and vascular complications. J Hypertens. 2012; 30(2): 375-383, doi: 10.1097/HJH.0b013e32834f04b6, indexed in Pubmed: 22179088.

21. Oudit GY, Kassiri Z, Jiang C, et al. SARS-coronavirus modulation of myocardial ACE2 expression and inflammation in patients with SARS. Eur J Clin Invest. 2009; 39(7): 618-625, doi: 10.1111/j.13652362.2009.02153.x, indexed in Pubmed: 19453650.

22. Lu R, Zhao X, Li J, et al. Genomic characterisation and epidemiology of 2019 novel coronavirus: implications for virus origins and receptor binding. Lancet. 2020; 395(10224): 565-574, doi: 10.1016/s0140-6736(20)30251-8.

23. Waxman DA, Kanzaria HK, Schriger DL. Acute myocardial infarction after laboratory-confirmed influenza infection. N Engl J Med. 2018; 378(26): 2538-2539, doi: 10.1056/NEJMc1805679, indexed in Pubmed: 29952170.

24. Shi S, Qin Mu, Shen Bo, et al. Association of cardiac injury with mortality in hospitalized patients with COVID-19 in Wuhan, 
China. JAMA Cardiol. 2020; 5(7): 802-810, doi: 10.1001/jamacardio.2020.0950, indexed in Pubmed: 32211816.

25. Luo W, Hong Y, Gou J, et al. Clinical pathology of critical patient with coronavirus pneumonia (COVID-19). Pre-Prints. 2020: $1-14$.

26. Wu Z, McGoogan JM. Characteristics of and Important Lessons From the Coronavirus Disease 2019 (COVID-19) Outbreak in China: Summary of a Report of 72314 Cases From the Chinese Center for Disease Control and Prevention. JAMA. 2020; 323(13): 1239-1242, doi: 10.1001/jama.2020.2648, indexed in Pubmed: 32091533.

27. Shi S, Qin Mu, Shen Bo, et al. Association of cardiac injury with mortality in hospitalized patients with COVID-19 in Wuhan, China. JAMA Cardiol. 2020; 5(7): 802-810, doi: 10.1001/jamacardio.2020.0950, indexed in Pubmed: 32211816.
28. Duffy EY, Cainzos-Achirica M, Michos ED. Primary and secondary prevention of cardiovascular disease in the era of the coronavirus pandemic. Circulation. 2020; 141(24): 1943-1945, doi: 10.1161/ CIRCULATIONAHA.120.047194, indexed in Pubmed: 32310675

29. Huang Z, Jiang $Y$, Chen J, et al. Inhibitors of the renin-angiotensin system: The potential role in the pathogenesis of COVID-19. Cardiol J. 2020; 27(2): 171-174, doi: 10.5603/CJ.a2020.0056, indexed in Pubmed: 32286678.

30. Kowalik MM, Trzonkowski P, Łasińska-Kowara M, et al. COVID-19 - Toward a comprehensive understanding of the disease. Cardiol J. 2020; 27(2): 99-114, doi: 10.5603/CJ.a2020. 0065, indexed in Pubmed: 32378729.

31. Dzieciatkowski T, Szarpak L, Filipiak KJ, et al. COVID-19 challenge for modern medicine. Cardiol J. 2020; 27(2): 175-183, doi: 10.5603/CJ.a2020.0055, indexed in Pubmed: 32286679. 